\title{
Erratum: Practical criteria for $R$-positive recurrence of unbounded semigroups*
}

\author{
Nicolas Champagnat $^{\dagger} \quad$ Denis Villemonais $^{\dagger}$
}

\begin{abstract}
The proof of the second convergence of Theorem 2.1 provided in the original paper relies on properties of the so called Q-process, which is not, in general, defined on the whole state space. Hence the inequality is only proved for some restricted sets of functions and measures. This weakness can be easily repaired and we provide here a proof of the inequality that applies in full generality.
\end{abstract}

Keywords: R-positivity; quasi-stationary distributions; mixing properties; Foster-Lyapunov criteria.

AMS MSC 2010: 60J05; 60J25; 47A35.

Submitted to ECP on March 4, 2020, final version accepted on March 9, 2020.

In Theorem 2.1 of [2], we claim that, under Assumption (G), there exist two constants $C^{\prime}>0$ and $\beta \in(0,1)$ such that, for all measurable functions $f: E \rightarrow \mathbb{R}$ satisfying $|f| \leq \psi_{1}$ and all positive measures $\mu$ on $E$ such that $\mu\left(\psi_{1}\right)<+\infty$,

$$
\left|\theta_{0}^{-n} \mu P_{n} f-\mu(\eta) \nu_{P}(f)\right| \leq C^{\prime} \beta^{n} \mu\left(\psi_{1}\right) .
$$

The proof provided in [2] is incomplete since it is based on properties of the so called $Q$-process, which is not, in general, defined on the whole state space $E$. The consequence is that the above inequality is only proved for functions $f$ that vanish when $\eta$ vanishes and for probability measures $\mu$ such that $\mu(\{x \in E, \eta(x)=0\})=0$.

We provide here a proof of the inequality that applies to all functions $f: E \rightarrow \mathbb{R}$ satisfying $|f| \leq \psi_{1}$ and all positive measures $\mu$ on $E$ such that $\mu\left(\psi_{1}\right)<+\infty$, as claimed in the original result.

Proof. Recall that the semi-group $\left(Q_{n}\right)_{n \in \mathbb{Z}_{+}}$, defined by

$$
Q_{n}(f)=\frac{P_{n}\left(f \psi_{1}\right)}{\left(\theta_{1}+c_{2}\right)^{n} \psi_{1}}, \forall n \geq 0,\|f\|_{\infty} \leq 1,
$$

satisfies conditions (E1-E4) of [1] with $\varphi_{1}=1$ and $\varphi_{2}=\psi_{2} / \psi_{1}$, using $\theta_{1} /\left(\theta_{1}+c_{2}\right)$ in place of $\theta_{1}, \theta_{2} /\left(\theta_{1}+c_{2}\right)$ in place of $\theta_{2}$ and $c_{1} /\left(\theta_{1}+c_{2}\right)^{n_{1}}$ in place of $c_{1}$. In particular, according to Theorem 2.1 in [1], there exist constants $C>0, \alpha \in(0,1)$ and a probability measure

\footnotetext{
${ }^{*}$ Main article: https://doi.org/10.1214/20-ECP288.

${ }^{\dagger}$ Université de Lorraine, CNRS, Inria, IECL, UMR 7502, F-54000 Nancy, France.

E-mail: nicolas . champagnat@inria.fr, denis.villemonais@univ-lorraine.fr
} 
$\nu_{Q S D}$ on $E$ such that, for all bounded measurable functions $g: E \rightarrow \mathbb{R}$ and all probability measures $v$ such that $v\left(\varphi_{2}\right)>0$,

$$
\left|\frac{v Q_{n} g}{v Q_{n} \mathbb{1}}-\nu_{Q S D}(g)\right| \leq C \alpha^{n} \frac{\|g\|_{\infty}}{v\left(\varphi_{2}\right)} .
$$

Multiplying both sides of the inequality by $v Q_{n} \mathbb{1}$ and applying the obtained inequality to $v=\left(\delta_{x}+\nu_{Q S D}\right) / 2$, one obtains, for all $x \in E$ (recall that $\nu_{Q S D}\left(\varphi_{2}\right)>0$ ),

$$
\left|Q_{n} g(x)+\nu_{Q S D} Q_{n} g-\nu_{Q S D}(g)\left(Q_{n} \mathbb{1}(x)+\nu_{Q S D} Q_{n} \mathbb{1}\right)\right| \leq 2 C \alpha^{n} \frac{\|g\|_{\infty}\left(Q_{n} \mathbb{1}(x)+\nu_{Q S D} Q_{n} \mathbb{1}\right)}{\varphi_{2}(x)+\nu_{Q S D}\left(\varphi_{2}\right)} .
$$

But $\nu_{Q S D}$ being a quasi-stationary distribution for $Q$, we have $\nu_{Q S D} Q_{n} g=\theta_{Q}^{n} \nu_{Q S D}(g)$ for some $\theta_{Q} \in(0,1]$, thus

$$
\left|Q_{n} g(x)-\nu_{Q S D}(g) Q_{n} \mathbb{1}(x)\right| \leq 2 C \alpha^{n} \frac{\|g\|_{\infty}\left(Q_{n} \mathbb{1}(x)+\nu_{Q S D} Q_{n} \mathbb{1}\right)}{\nu_{Q S D}\left(\varphi_{2}\right)} .
$$

Theorem 2.5 of [1] for $Q_{n}$ states that there exists a function $\eta_{Q} \in L^{\infty}$ such that

$$
\left|\theta_{Q}^{-n} Q_{n} \mathbb{1}(x)-\eta_{Q}(x)\right| \leq C^{\prime \prime} \alpha^{\prime n},
$$

for some constants $\alpha^{\prime} \in(0,1)$ and $C^{\prime \prime} \geq 0$. We deduce that

$$
\begin{aligned}
\left|\theta_{Q}^{-n} Q_{n} g(x)-\nu_{Q S D}(g) \theta_{Q}^{-n} Q_{n} \mathbb{1}(x)\right| & \leq 2 C \alpha^{n} \frac{\|g\|_{\infty}\left(\theta_{Q}^{-n} Q_{n} \mathbb{1}(x)+\theta_{Q}^{-n} \nu_{Q S D} Q_{n} \mathbb{1}\right)}{\nu_{Q S D}\left(\varphi_{2}\right)} \\
& \leq 2 C \alpha^{n} \frac{\|g\|_{\infty}\left(C^{\prime \prime}+C^{\prime \prime}\left\|\eta_{Q}\right\|_{\infty}+1\right)}{\nu_{Q S D}\left(\varphi_{2}\right)}
\end{aligned}
$$

and hence that

$$
\left|\theta_{Q}^{-n} Q_{n} g(x)-\nu_{Q S D}(g) \eta_{Q}(x)\right| \leq 2 C \alpha^{n} \frac{\|g\|_{\infty}\left(C^{\prime \prime}+C^{\prime \prime}\left\|\eta_{Q}\right\|_{\infty}+1\right)}{\nu_{Q S D}\left(\varphi_{2}\right)}+\nu_{Q S D}(g) C^{\prime \prime} \alpha^{\prime n} .
$$

Multiplying both sides by $\psi_{1}(x)$, setting $f=g \psi_{1}$ and integrating with respect to $\mu(\mathrm{d} x)$ ends the proof.

\section{References}

[1] Nicolas Champagnat and Denis Villemonais, General criteria for the study of quasi-stationarity, arXiv e-prints (2017), arXiv:1712.08092.

[2] Nicolas Champagnat and Denis Villemonais, Practical criteria for $R$-positive recurrence of unbounded semigroups, Electron. Commun. Probab. 25 (2020), 11 pp. 